* D. Cortina-Gil ${ }^{1}$ and $\mathbf{W}$. Mittig ${ }^{2}$

* ' Universidad de Santiago de Compostela, 15786 Santiago de Compostela, Spain

* ${ }^{2}$ Michigan State University and National Superconducting Laborator, East Lansing, Michigan 48824, USA

DOI: $10.1051 /$ epn/2010204

\title{
PROBING NUCLEAR FORCES AT THE EXTREME OF ISOSPIN:
}

\section{THE ${ }^{7} \mathrm{H}$ RESONANCE}

Modern high-intensity accelerators provide access to new regions of the nuclear chart. This allows one to study the properties of extremely weakly bound or even unbound nuclei which spontaneously emit protons or neutrons. These "exotic" nuclei have a huge imbalance in the proton/neutron ratio, adding a new degree of freedom - the isospin - and opening a large field of new experimental possibilities.

$\Delta$ An inner

thas been observed that, in these systems, nuclear forces manifest themselves in different ways. This is interpreted presently as an isospin dependence, and is questioning our understanding of the behaviour of nuclear matter going from proton- rich to neutron-rich matter.

The description of nuclear forces in these weakly bound nuclear systems represents an enormous challenge. The interaction between nucleons is responsible for the creation of particular structures. The study of these structures, at the limit of nuclear binding and beyond, where non-perturbative phenomena become important, is a unique source of information. "Ab initio" calculations illustrate nicely these non-perturbative phenomena. These calculations start from interactions describing perfectly a large body of nucleon-nucleon scattering data and then solve, without further approximation, the nuclear n-body problem. However, they fail completely in reproducing macroscopic observables as, for example, nuclear binding energies (see figure 1) [1]. These large discrepancies are attributed to the importance of 3-body forces: the interaction between two nucleons is modified by the presence of a third nucleon.

\section{Non-perturbative phenomena in nuclear physics}

We can explain nuclear interaction in terms of virtual pion exchange between two nucleons. This exchange may, for example, result in an excitation of the $\Delta$-resonance [unstable nuclear state defined by a mean energy value $\left(E_{R}\right)$ and a given width $\left(\Gamma_{R}\right)$, determined by its half-life $(\mathrm{dt})$ due to the relation $\Gamma_{\mathrm{R}} \cdot \mathrm{dt}=\hbar$ ] of one of the nucleons that interacts in this excited state with a $3^{\text {rd }}$ nucleon present in the nuclei. This last interaction can be seen as a higher order correction of the original interaction of the first two nucleons. The importance of this higher order correction is not due to the strength associated with the interaction but to its intrinsic nature. Properties of very strong Coulomb interaction have been tested in the Mott-scattering of $\mathrm{Pb}+\mathrm{Pb}[2]$. The Coulomb potential energy in this system is of the order of $500 \mathrm{MeV}$, one order of magnitude higher than the binding energy of the systems of figure 1. However, in this particular case higher order corrections, such as vacuum polarization, are only of the order of $10^{-4}$. So in the case of the nuclear interaction, the origin of the 
III strong effect of higher order corrections cannot be attributed to the interaction strength, but is due to the fact that it involves the strong interaction that has intrinsically non-perturbative aspects.

The coupling to continuum in loosely bound or unbound systems becomes so important that it cannot be treated as a perturbation. Examples of a non-pertubative treatment have been achieved in recent shell-model calculations [3].

We focus now on another example of non-perturbative systems: the creation of very asymmetric nuclear matter and, particularly, on the observation of the extremely neutron-rich hydrogen isotope ${ }^{7} \mathrm{H}$, that emits spontaneously 4 neutrons leading to a triton $\left({ }^{3} \mathrm{H}\right)$. The search and characterization of Hydrogen resonances is one of the stringent tests about nuclear formation. Recent theoretical predictions [4] along with experimental

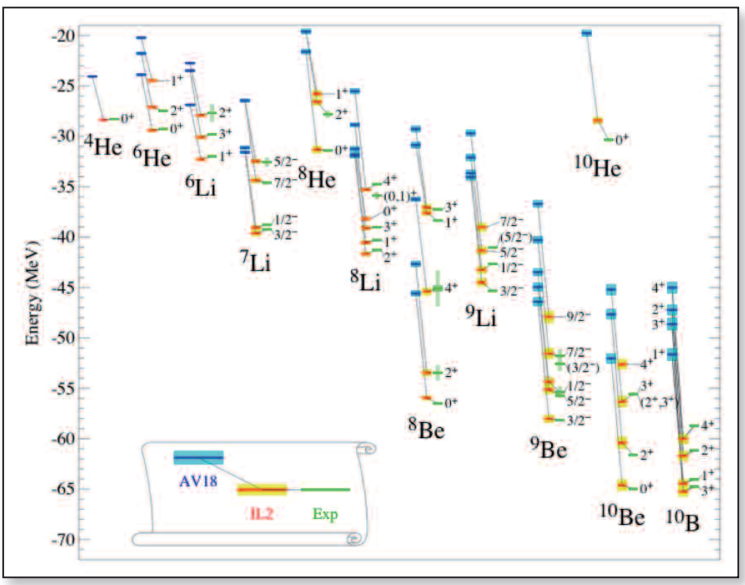

$\Delta$ FIG. 1: Comparison of experimental binding energies (green) for lower quantum states of light nuclear systems $A=4-10$ with those predicted by Greens function Monte Carlo GFMC method [1]. These predictions are built first using a realistic two-body interaction, the Argonne V18 (AV18) potential (blue), and then the Illinois-2 (IL2) three-nucleon potentia I(red). The use of three-nucleon potential improves notably the result, suggesting the importance of using a more and more complete description of the nuclear force. Thin black lines are used to connect predictions and experimental values for each state. Credits for the figure to S.C Pieper, R. Wiringa and J.Carlson.

V FIG. 2: The active target MAYA with its different components: active volume and amplification/detection zone. This picture illustrates a typical example of a binary reaction in the gas target. The secondary beam (red horizontal line) enters into the gas cell producing a binary reaction (vertex). This results in a fragment (black line) that is stopped inside the gas volume and a light charge fragment (blue line) with higher energy that escapes and is further identified in an ancillary detector (Csl scintillation crystal wall).

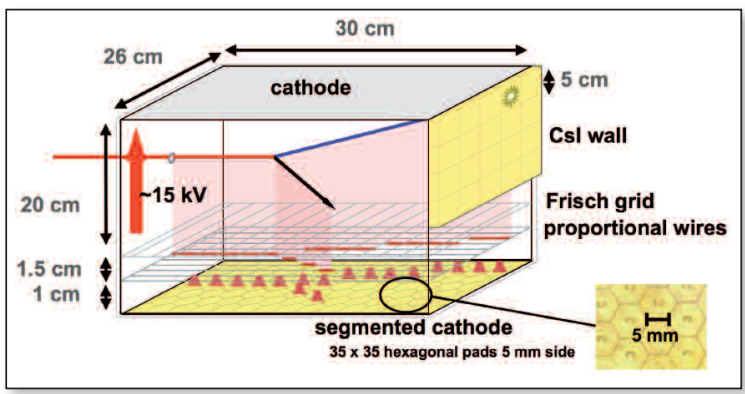

indications [5] predict that ${ }^{7} \mathrm{H}$ with six neutrons and a single proton will be the heaviest possible hydrogen system and will also constitute the most exotic nuclear system ever synthesized with a N/Z=6.

\section{Study of very exotic nuclear systems}

The synthesis and study of exotic nuclear systems require the use of radioactive beam facilities that provide beams of unstable nuclei, not existing on earth, from intense stable beams. The Isotope Separation On Line (ISOL) method is used for the production of lowenergy unstable beams. The primary stable beam impinges on a thick target where the radioactive species are created. They need to be extracted and accelerated up to energies suitable for studying nuclear reactions around the Coulomb barrier and higher.

The relatively low intensities of the exotic beam produced, together with the extremely low cross-sections of the reactions involved make the experimentation with these exotic nuclei very challenging. Fortunately, the sensitivity of the detectors and of the experimental methods used has impressively increased in the last years allowing nowadays detailed spectroscopic studies with beam intensities down to the order of 1 particle/s compared with values of $5.10^{9}$ particles/s that were necessary two decades ago.

The use of detection systems with the highest possible efficiency is essential. Another option is the increase of the target thickness, but this dramatically reduces the energy resolution achieved and compromises the information obtained

An elegant solution is found in the use of active targets: gaseous detectors where the filling gas plays the dual role of detection gas and target. They compensate for the low rates associated with exotic nuclei by achieving a large detection efficiency, which can nominally reach $100 \%$. Further, they can determine the interaction point between projectile and target nuclei on an event-by-event basis, allowing the gas target thickness to be increased without degrading the energy resolution. Moreover, the detection of recoils inside the active target reduces the detection threshold to a minimum, and thus guarantees detection even for very low recoil energies.

This new approach has been used in the experimental study of the ${ }^{7} \mathrm{H}$ system [6], performed at GANIL (France), where a secondary beam of ${ }^{8} \mathrm{He}$ with an energy of $15.4 \mathrm{MeV}$ per nucleon was produced in the Spiral facility by the Isotope Separation On Line (ISOL) technique [8].

The experimental detection setup consisted mainly of the active target MAYA [9] depicted in figure 2, working essentially like an ionization chamber. The MAYA detector is divided into two working zones. The ionisation zone (upper part) consists of an active volume $\left(26 \times 20 \times 30 \mathrm{~cm}^{3}\right)$ filled with isobutane $\mathrm{C}_{4} \mathrm{H}_{10}$. The amplification/detection 
zone (lower part) contains a wire plane and a cathode plane segmented in $35 \times 35$ hexagonal pads ( $5 \mathrm{~mm}$ side). The ${ }^{7} \mathrm{H}$ system was produced by the one-proton transfer reaction of the ${ }^{8} \mathrm{He}$ beam with the carbon atoms in the isobutane, ${ }^{8} \mathrm{He}+{ }^{12} \mathrm{C} \rightarrow{ }^{7} \mathrm{H}+{ }^{13} \mathrm{~N}$. Beam particles and charged reaction products ionize the isobutane along their paths in the gas. The electrons released in the ionization process drift toward the amplification zone, where they are multiplied around the wires inducing a mirror charge in the pads of the segmented cathode. The determination of the drift time and of the charge collected on the pads provides, in principle, the 3-D reconstruction of the trajectories of the secondary beam $\left({ }^{8} \mathrm{He}\right)$ and of the reaction products $\left({ }^{7} \mathrm{H}\right.$ and $\left.{ }^{13} \mathrm{~N}\right)$. Figure 3 presents typical examples of trajectory imaging with MAYA. In figure 3 (left panel), the image of the study of the binary ${ }^{11} \mathrm{Li}+\mathrm{p} \rightarrow{ }^{9} \mathrm{Li}+\mathrm{t}$ reaction [10] is shown. In this case we can identify clearly the trajectory of the exotic-projectile $\left({ }^{11} \mathrm{Li}\right)$ and both reaction products ( ${ }^{9} \mathrm{Li}$ and triton).

Figure 3 (right panel) depicts the ${ }^{8} \mathrm{He}+{ }^{12} \mathrm{C} \rightarrow{ }^{7} \mathrm{H}+{ }^{13} \mathrm{~N}$ reaction, where only ${ }^{13} \mathrm{~N}$ is registered in the active target volume. The ${ }^{7} \mathrm{H}$ identification is more complex due to its unstable nature. The scattered ${ }^{7} \mathrm{H}$ decays very rapidly, before being detected, into triton and four neutrons $\left({ }^{7} \mathrm{H} \rightarrow \mathrm{t}+4 \mathrm{n}\right)$. The experimental setup was not equipped for the detection of neutrons, thus the reaction channel is determined by detecting the remaining charged particles, triton and nitrogen, that could not be simultaneously stopped within the MAYA active volume.due to their very different kinematical properties.

The gas pressure was thus adjusted to stop ${ }^{13} \mathrm{~N}$, and secondary beam particles ${ }^{8} \mathrm{He}$ and tritons, with too low energy-loss in the active volume, could not be traced. Tritons escaped the active volume and were detected by a segmented wall of CsI crystals covering the exit face of MAYA(see figure 2).

The charge image induced by the recoil nitrogen isotopes was projected in the segmented cathode, where the charge was collected and the range and the total charge determined. The coincident detection of triton and nitrogen restricted the selection to such a binary process and discards contamination from other reaction channels. The recoil scattering angle was determined too, and together with the 3-D tracking capabilities of MAYA allows the extraction of the complete kinematics of the nitrogen selected.

Our experimental setup did not allow an isotopic separation of the recoiling nitrogen. Different reaction channels leading to triton and a recoiling nitrogen isotope are separated using their different kinematics. The points displayed in figure 4 correspond to different nitrogen isotopes that survive the stringent analysis filters. They originate from a binary reaction and are in coincidence with a triton detected in the CsI(Tl) wall. The experimental data of figure 4 are compared to the

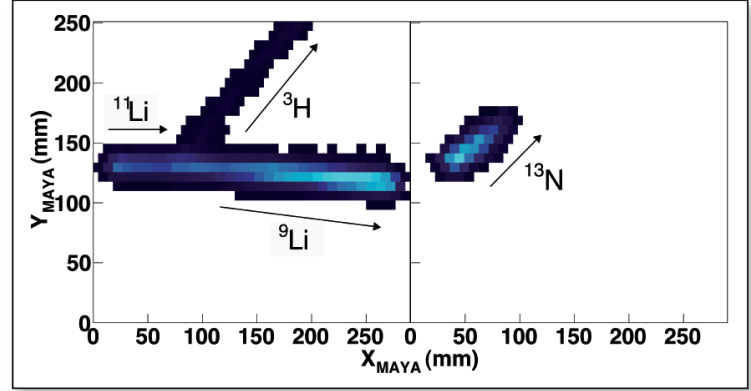

$\Delta$ FIG. 3: Example of measured binary reactions with MAYA. $X_{\text {MAYA }}$ and $Y_{\text {MAYA }}$ axis correspond to the dimensions of the segmented cathode $(26 \times 30 \mathrm{~cm})$ and show a projection of the reaction on the cathode plane. Left: Illustration of an event corresponding to the ${ }^{11} \mathrm{Li}+\mathrm{p} \rightarrow{ }^{9} \mathrm{Li}+\mathrm{t}$ reaction [10]). Right: Illustration of an event corresponding to the ${ }^{8} \mathrm{He}+{ }^{12} \mathrm{C} \rightarrow{ }^{7} \mathrm{H}+{ }^{13} \mathrm{~N}$ reaction at $\sim 123 \mathrm{MeV}$ [7].

V FIG. 4: Kinematics of the different reaction channels ${ }^{8} \mathrm{He}+{ }^{12} \mathrm{C} \rightarrow{ }^{7} \mathrm{H}+{ }^{13} \mathrm{~N}$ red line, ${ }^{8} \mathrm{He}+{ }^{12} \mathrm{C} \rightarrow{ }^{6} \mathrm{H}+{ }^{14} \mathrm{~N}$ black line and ${ }^{8} \mathrm{He}+{ }^{12} \mathrm{C} \rightarrow{ }^{5} \mathrm{H}+{ }^{15} \mathrm{~N}$ blue line, with all the experimental data that pass all the analysis filters. The data points marked in red are associated to ${ }^{13} \mathrm{~N}$ and directly connected with the ${ }^{7} \mathrm{H}$ resonance formation. Different size boxes represent the experimental error associated with the ${ }^{13} \mathrm{~N}$ energy and angle determination for each particular event. The black data points correspond to heavier $\mathrm{N}$ isotopes and are linked to formation of lighter $\mathrm{H}$ isotopes.

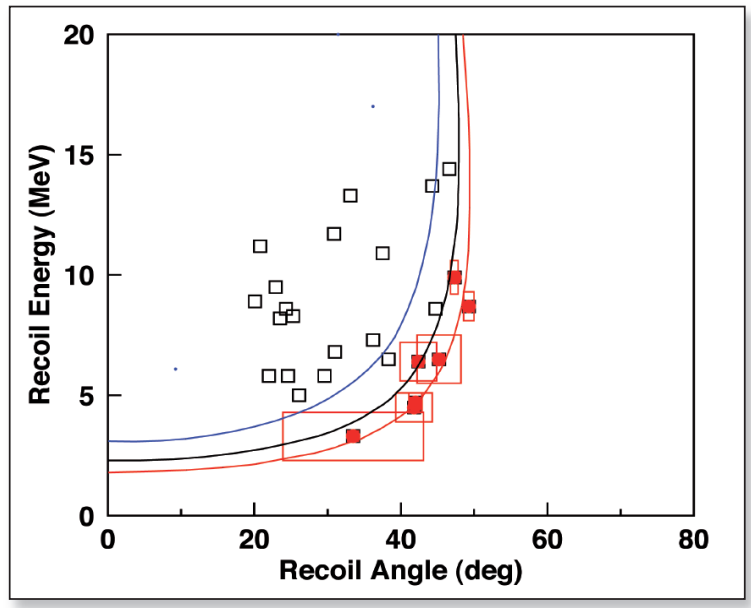

kinematics of ${ }^{7} \mathrm{H}$ formation assuming a rest mass of ${ }^{7} \mathrm{H}$ system equal to the triton and four neutrons (red curve). The same is applied to calculate the kinematics for the ${ }^{6} \mathrm{H}$ (black curve) and ${ }^{5} \mathrm{H}$ (blue curve) formation. Several events (red squares in figure 4) accumulate clearly around the kinematics line corresponding to the production of ${ }^{7} \mathrm{H}$. The boxes surrounding each event represent experimental errors associated with the determination of the recoil energy and deflecting angle. The ${ }^{7} \mathrm{H}$ resonance energy $\mathrm{E}_{\text {exc }}$, defined as the energy above the threshold for the decay of ${ }^{7} \mathrm{H} \rightarrow \mathrm{t}+4 \mathrm{n}$, is shown in figure 5 (black histogram). This distribution is normalised by the corresponding measured cross section, which resulted in a (d $\sigma / \mathrm{d} \omega)$ of $40.1 \mu \mathrm{b} / \mathrm{sr}$, with an upper limit fixed by the experimental uncertainty of 98.1 and lower limit of $9.5 \mu \mathrm{b} / \mathrm{sr}$. The distribution is then described with a modified Breit-Wigner function, where the cross section depends on the excitation energy through the resonance energy $\left(E_{R}\right)$ and width $(\Gamma)$. 
These parameters are extracted, following a multiparametric Maximum Likelihood fit procedure which is especially suited for low statistical samples. The result is a narrow resonance (yellow function in figure 5) with a width $(\Gamma)$ of $0.09 \mathrm{MeV}$ (1.03 and $0.03 \mathrm{MeV}$ upper and lower limit) and a resonance energy $\left(E_{R}\right)$ of $0.57 \mathrm{MeV}$ (0.99 and 0.36 upper and lower limit), located just above the threshold of the $t+4 n$ sub-system.

The width of a resonance is directly related to the lifetime of the resonant state. In figure 6 , the width expected for uncorrelated four neutron decay as calculated by Golovkov et al. [11] is shown for two different parametrisations, together with the experimental value extracted from this analysis. The much larger experimental width implies a correlation of the four neutrons in the emission process. This may be interpreted as a hint for the emission of a neutron cluster, that speculatively may be related to present discussions about the existence of a tetra-neutron

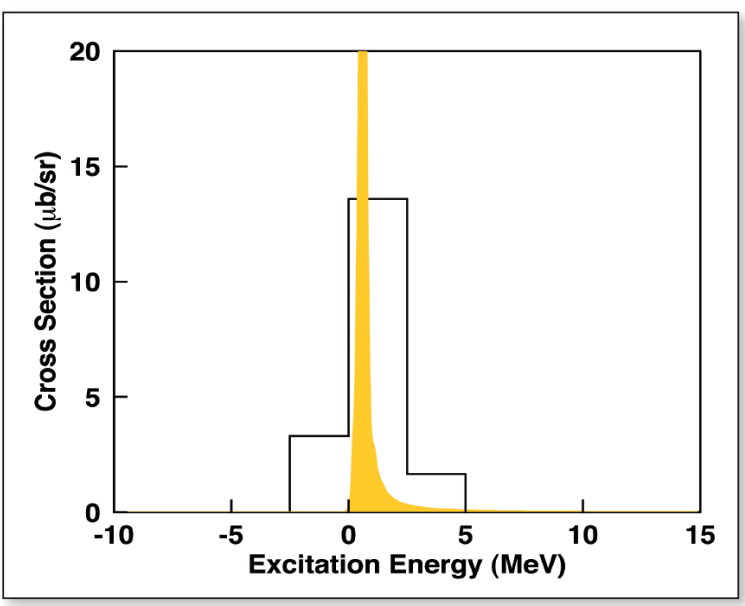

A FIG. 5: Energy spectra above the separation threshold of ${ }^{7} \mathrm{H} \rightarrow \mathbf{t}+4 \mathrm{n}$ normalised to the measured cross-section for the events identified as ${ }^{7} \mathrm{H}$. The histogram (black solid line) corresponds to the experimental measurement whereas the yellow function is the modified Breit-Wigner function fitted to allow the characterization of the resonance parameters.

V FIG. 6: Decay width $\Gamma$ for the ${ }^{7} \mathrm{H}$ system for an uncorrelated emission of triton and four neutrons evaluated by Golovkov et al. [11] using hyperspherical coordinates and for two different values of the k parameter related with the radial part of the interaction (green and blue curves), together with the experimental result.

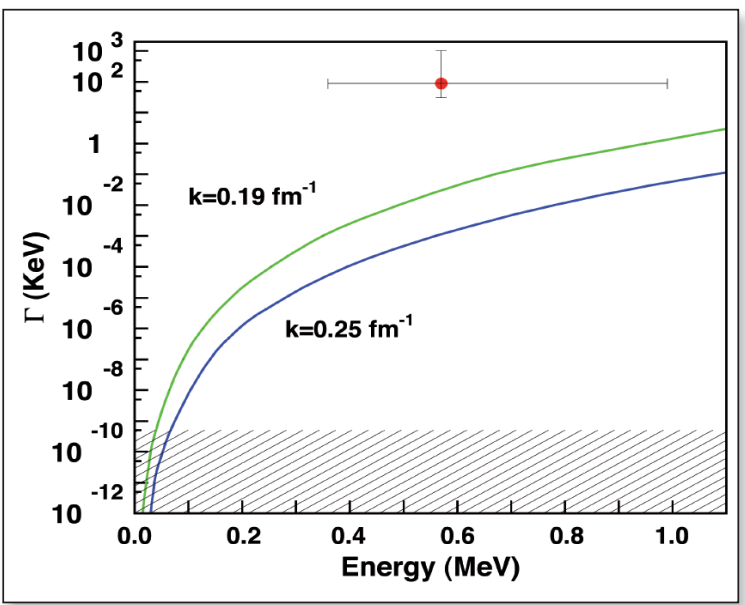

state [12]. This effect can be seen as another illustration of the non-pertubative character of such a system near threshold, where special cluster structures may play an important role.

To conclude, the existence of a ${ }^{7} \mathrm{H}$ system formed as a resonance above the threshold of the decay in a multibody system consisting of four neutrons and one triton has been observed and characterized. This was possible only by the use of a new generation high sensitivity active target detector. The non-perturbative description of the decay of such systems is without any doubt a major challenge for present theories.

\section{About the authors}

Dolores Cortina-Gil (Docteur en Sciences of the University of Caen, France 1996) started her scientific activity in GANIL, France and GSI, Germany. In 2000 she joint the University of Santiago de Compostela, Spain, where she is nowadays Professor at the Particle Physics Department.

Wolfgang Mittig (Docteur en Sciences of the University of Paris, France 1971) was researcher in Commisariat de l'Energie Atomique, CEA, France. For 5 years he was member of the University of Sao Paolo, Brazil and did his "livre docente" thesis there. In 1982 he became Staff Scientist of GANIL, France, as member of the CEA. Since January 2008 he is Hannah Professor at MSUNSCL, MI, East Lansing,USA.

\section{Aknowledgments}

The experimental work presented here has been performed in the framework of an international collaboration and is part of the $\mathrm{PhD}$ of M. Caamaño. Authors want to thank M. Caamaño and T. Roger for their invaluable help preparing figures.

\section{References}

[1] S.C. Pieper, R.B. Wiringa and J. Carlson, Physical Review C 70 (2004) 054325, and private communication

[2] A.C.C. Villari et al., Phys. Rev. Lett. 71 (1993) 2551

[3] N. Michel, W. Nazarewicz, M. Ploszajczak and K. Nennaceur, Phys. Rev. Lett. 89, (2002) 042502; A.Volya and V. Zelevinsky, Phys. Rev. C 74 (2006) 064314

[4] S. Aoyama and N. Itagaki, Nucl. Phys. A 738 (2004) 362.

[5] A.A. Korsheninnikov et al., Phys. Rev. Lett. 90 (2003), 082501.

[6] N. K. Timofeyuk, Phys. Rev. C 65 (2002), 064306.

[7] M. Caamaño et al., Phys. Rev. Lett. 99 (2007) 062502

[8] www.ganil.fr

[9] C.E. Demonchy et al., Nucl. Inst. \& Meth. A 583 (2007) 341

[10] T. Roger et al., Phys Rev C 79 (R) (2009) 0316003, I.Tanihata et al., Phys. Rev. Lett. 100 (2008) 192502

[11] M.S. Golovkov et al., Phys. Lett. B 588 (2004) 163

[12] S.C. Pieper, Phys. Rev. Lett. 90 (2003) 252501, and references therein 